\title{
Species Identification of the Tropical Abalone (Haliotis asinina, Haliotis ovina, and Haliotis varia) in Thailand Using RAPD and SCAR Markers
}

\author{
Sirawut Klinbunga ${ }^{\dagger} *$, Piti Amparyup ${ }^{\ddagger}$, Rungnapa Leelatanawit ${ }^{\ddagger}$, Anchalee Tassanakajon ${ }^{\S}$, \\ Ikuo Hirono", Takashi Aoki", Padermsak Jarayabhand ${ }^{\#, * *}$ and Piamsak Menasveta ${ }^{\dagger, * *}$ \\ ${ }^{\dagger}$ Marine Biotechnology Research Unit, National Center for Genetic Engineering and Biotechnology (BIOTEC), National Science and \\ Technology Development Agency (NSTDA), 113 Paholyothin Rd., Klong 1, Klong Luang, Pathumthani 12120 Thailand \\ ${ }^{\ddagger}$ Program of Biotechnology, ${ }^{\S}$ Department of Biochemistry, \\ **Department of Marine Science, Faculty of Science, Chulalongkorn University, Bangkok 10330, Thailand \\ "Laboratory of Genome Science, Graduate School of Marine Science and Technology, \\ Tokyo University of Marine Science and Technology, Konan 4-5-7, Minato-ku, Tokyo 108-8477, Japan \\ "Aquatic Resources Research Institute, Chulalongkorn University, Bangkok, 10330, Thailand
}

Received 12 May 2003, Accepted 7 July 2003

\begin{abstract}
A randomly amplified polymorphic DNA (RAPD) analysis was used to identify the species- and population-specific markers of abalone; Haliotis asinina, $\mathrm{H}$. ovina, and $\mathrm{H}$. varia in Thai waters. Fifteen species-specific and six populationspecific RAPD markers were identified. In addition, an 1650 bp band (UBC195) that was restricted to $H$. ovina from the Gulf of Thailand (east) was also found. All of the specific RAPD markers were cloned and sequenced. Twenty pairs of primers were designed and specificitytested $(N=12$ and 4 for target and non-target species, respectively). Seven primer pairs (CUHA1, 2, 4, 11, 12, 13, and 14) were specifically amplified by $H$. asinina DNA, whereas a single pair of primers showed specificity with $H$. ovina (CUHO3) and $H$. varia (CUHV1), respectively. Four primer pairs, including CUHA2, CUHA12, CUHO3, and CUHV1, were further examined against 216 individuals of abalone $(N=111,73$, and 32 , respectively $)$. Results indicated the species-specific nature of all of them, except CUHO3, with the sensitivity of detection of $100 \mathrm{pg}$ and 20 pg of the target DNA template for CUHA2 and CUHA12 and CUHV1, respectively. The species-origin of the frozen, ethanol-preserved, dried, and boiled $H$. asinina specimens could also be successfully identified by CUHA2.
\end{abstract}

Keywords: Abalone, PCR, RAPD, SCARs, Species-specific markers

\footnotetext{
*To whom correspondence should be addressed.

Tel: 66-2-2185279; Fax: 66-2-2547680

E-mail: sirawut@biotec.or.th
}

\section{Introduction}

Abalones are economically important archeogastropods that are currently cultured worldwide. There are over 15 species of abalone, which are being farmed and are commercially important (Jarayabhand and Paphavasit, 1996). The total world production of abalone was approximately 13,000 metric tons in $1999,7,165$ tons of which $(55 \%)$ were produced on farms. The major producers of abalone are China and Taiwan. They annually contribute approximately $75 \%$ of the cultured production (Gordon, 2000).

Three species of tropical abalone (Haliotis asinina, $H$. ovina, and H. varia) are found in Thai waters (Jarayabhand and Paphavasit, 1996). Both $H$. asinina and $H$. ovina are distributed along the east coast of the upper Gulf of Thailand, and all three species occur in the Andaman Sea (Tookvinas et al., 1986; Nateewatana and Bussarawit, 1988). Among these abalone, $H$. asinina, provides the highest percentage $(85 \%)$ between the meat weight and total weight when compared to $H$. ovina (40\%) and $H$. varia (30\%), respectively (Singhagraiwan and Doi, 1993). Accordingly, H. asinina is presently being initially commercially cultured in Thailand.

Appropriate genetic markers can be used to elevate the cultural and management efficiency of abalone in Thailand (Jarayabhand et al., 2002). The sustainable success of aquacultural activity of commercially important species requires a basic knowledge on stock structure as well as the use of suitable molecular genetic markers to establish broodstock management programs of exploited species (Avise, 1994; Calvalho and Hauser, 1994). In addition, species-specific markers play important roles in preventing the supply of incorrect abalone larvae for the industry as well as 
quality control of abalone from Thailand. These markers are also necessary for the development of monospecific farming of $H$. asinina in Thailand.

Species-specific sequences of lysin were reported in the pink $(H$. corrugata) and red $(H$. rufescens) abalone (Vacquier et al., 1990). Partial sequences of $18 \mathrm{~S}$ rDNA were also used to differentiate the closely related abalone, $H$. discus discus and $H$. discus hannai. The amplified 18S rDNA was directly sequenced and multiple-aligned with that of $H$. madaka, $H$. gigantea, and a land gastropod (Limicolaria kambeul). The inferred 18S rDNA phylogeny indicated that $H$. discus discus and $H$. discus hannai are closely related but distinguishable at the subspecies level (Naganuma et al., 1998). In addition, a tandemly-repeated satellite DNA (290-291 bp in length) was identified by Sal I digestion of genomic DNA of five Eastern Pacific (California) abalone species, including the red $(H$. rufescens), white (H. sorenseni), flat (H. walallensis), pinto (H. kamtschatkana), and pink (H. corrugata) abalone. Sequences of satellite DNA were determined by direct sequencing and revealed species-specificity in these abalone (Muchmore et al., 1998). For identification of the species origins of abalone tissue from South Africa, species-specific PCR was developed to distinguish $H$. midae from $H$. spadicea using a portion of lysin cDNA sequences (Lee and Vacquier, 1995). PCR specifically amplified a $1,300 \mathrm{bp}$ fragment of the genomic DNA from dried, cooked, and fresh $H$. midae tissue. A smaller fragment of the $146 \mathrm{bp}$ product was successfully amplified and used for the identification of canned $H$. midae. Additionally, PCR-RFLP revealed interspecific polymorphism that differentiated these species unambiguously (Sweijd et al., 1998).

Recently, species-diagnostic markers of $H$. asinina, $H$. ovina, and $H$. varia were developed using PCR-RFLP of 16S rDNA (Jarayabhand et al., 2002). Restriction of the amplified $16 \mathrm{~S}$ rDNA with Alu I could differentiate these abalone unambiguously (patterns $\mathrm{A}$ and $\mathrm{E}$ in $H$. asinina, $N=115$; $\mathrm{B}$ in H. ovina, $N=71$ and $\mathrm{C}, \mathrm{D}, \mathrm{F}$, and $\mathrm{G}$ in $H$. varia, $N=23$ ). Further digestion of $16 \mathrm{~S}$ rDNA with Bam HI, Eco RI, and Hae III yielded non-overlapping composite haplotypes in these abalones; AAAA and AAAE in $H$. asinina, ABBB, $\mathrm{AAAB}$ and $\mathrm{AABB}$ in $H$. ovina and $\mathrm{BABG}, \mathrm{BABC}, \mathrm{BABD}$, $\mathrm{BABF}$ and $\mathrm{AABG}$ in $H$. varia, respectively. Species-specific PCR, based on 16S rDNA polymorphism, was successfully developed in $H$. asinina and $H$. varia $(100 \%$ amplification success with no false positive) but not in H. ovina $(68.75 \%$ amplification success with extensive false positives from other species) (Klinbunga et al., 2003).

Interspecific hybridization and gene introgression between $H$. rubra and $H$. laevigata was reported, based on an allozyme analysis (Brown, 1995). Theoretically, hybridization between the male $H$. asinina and/or $H$. varia with females of different species could have eliminated the accuracy of the species identification, based on matriarchally inherited markers like 16S rDNA. As a result, $H$. asinina- and $H$. varia-specific nuclear DNA markers need to be developed.
Randomly amplified polymorphic DNA-polymerase chain reaction (RAPD-PCR) is a relatively simple method for the identification of genetic markers at different taxonomic levels, without the need for knowledge of sequences of the genome under investigation (Welsh and McClelland, 1990; Williams et al., 1990; Hadrys et al., 1992). The objective of this study was to develop reproducible species-specific markers of $H$. asinina in Thailand. Candidate species- and population-specific RAPD markers were cloned and sequenced. The primers were designed. Specificity, sensitivity, and stability of the selected markers were examined. The sequence-characterized amplified region (SCAR) markers that offered an accurate discrimination of $H$. asinina from $H$. ovina and $H$. varia were developed.

\section{Materials and Methods}

Sampling Specimens of three abalone species $[H$. asinina $(N=111), H$. ovina $(N=73)$, and $H$. varia $(N=32)]$ were collected (Table 1 and Fig. 1) and individually kept at $-30^{\circ} \mathrm{C}$ until required. Alternatively, the foot muscle of each specimen was dissected and kept at $-80^{\circ} \mathrm{C}$ until further use for the DNA extraction.

DNA Extraction The total DNA was extracted from a piece of the foot muscle of each abalone using the phenol-chloroformproteinase K method (Klinbunga et al., 1996). The concentration of the extracted DNA was spectrophotometrically estimated. The DNA was stored at $4^{\circ} \mathrm{C}$ until needed.

RAPD-PCR One hundred and thirteen primers that were purchased from Operon Technologies Inc. (Alameda, USA) and the University of British Columbia (Canada) were screened for amplification success against 2-3 representatives of each abalone species (Fritsch et al., 1993). Five primers (OPB11, UBC101, UBC195, UBC197, and UBC271) were tested against different populations of $H$. asinina ( $N=5$ per location), $H$. ovina $(N=7-8$ per location), and $H$. varia $(N=15$ and 3 for HVPHUW and HVPHAW).

RAPD-PCR was carried out in a $25 \mu \mathrm{l}$ reaction volume containing $10 \mathrm{mM}$ Tris- $\mathrm{HCl}, \mathrm{pH} 8.3,50 \mathrm{mM} \mathrm{KCl}, 0.001 \%$ gelatin, 2-3 mM of $\mathrm{MgCl}_{2}, 100 \mu \mathrm{M}$ of each dNTP, $0.2 \mu \mathrm{M}$ of a primer, 1 unit of AmpliTaq DNA Polymerase (Perkin-Elmer, Cetus, USA), and $25 \mathrm{ng}$ of a DNA template. The amplification was performed in an Omnigene thermal cycler (Hybaid, Middlesex, UK) according to Klinbunga et al. (2001). Five microlitres of the amplification reaction were electrophoresed through $1.6 \%$ agarose gels and visualized under a UV transilluminator after ethidium bromide staining (Maniatis et al., 1982).

Cloning and sequencing of abalone RAPD markers Twentytwo RAPD fragments, showing population-, region-, or speciesspecificity for each abalone (Table 2), were gel-eluted using a PrepA-Gene DNA purification kit (Bio-Rad Ltd, Hercules, USA) and reamplified with the original primer. The target band was eluted and ligated to the pGEM-T Easy vector (Promega, Madison, USA). One-tenth of the volume of each ligation reaction was 
Table 1. Sample collection sites and sample sizes of abalone (H. asinina, H. ovina and H. varia) used in this study

\begin{tabular}{llc}
\hline \multicolumn{1}{c}{ Geographic origin } & Abbreviation & Sample size $(N)$ \\
\hline H. asinina & & 10 \\
Samet Island, Rayong (Gulf of Thailand) & HASAME & 15 \\
P $_{0}$ stock, Rayong (Gulf of Thailand) & HARAYE & 20 \\
Cambodia (east of peninsular Thailand) & HACAME & 12 \\
P $_{0}$ stock, Cambodia (east of peninsular Thailand) & HACAMHE & 23 \\
Talibong Island, Trang (Andaman Sea) & HATRAW & 19 \\
F $_{1}$ Philippines & HAPHIE & 12 \\
Indonesia HAINDW & \\
H. Sina & & 20 \\
Sichang Island, Chon Buri (Gulf of Thailand) & HOCHOE & 19 \\
Samet Island, Rayong (Gulf of Thailand) & HOSAME & 18 \\
Churk Island, Trang (Andaman Sea) & HOTRAW & 16 \\
Similan Island, Phangnga (Andaman Sea) & HOPHAW & 29 \\
Haria & & 3 \\
L-Island, Phuket (Andaman Sea) & HVPHUW & 216 \\
Similan Island, Phangnga (Andaman Sea) & HVPHAW & \\
\hline Total ( $N$ ) & & \\
\hline
\end{tabular}

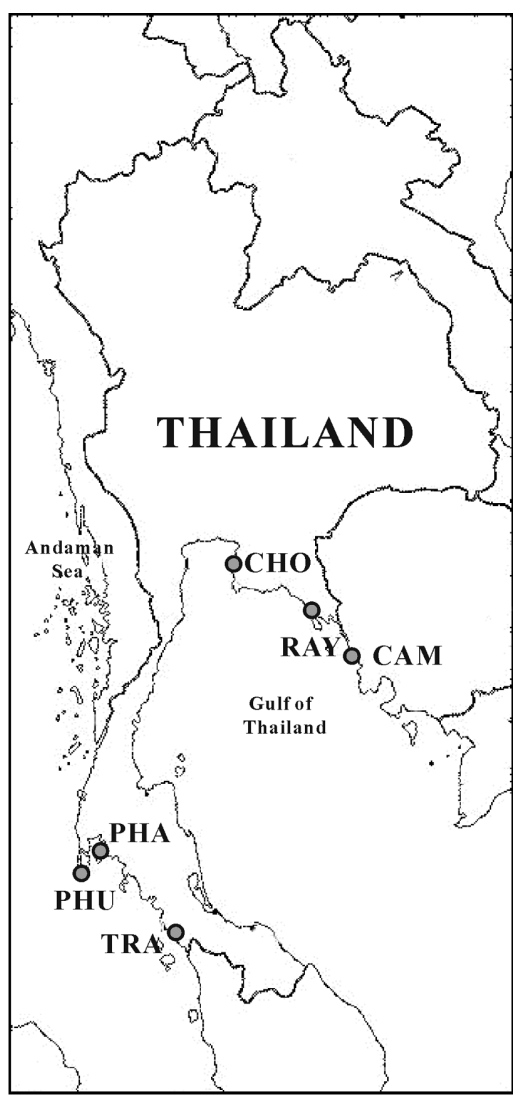

Fig. 1. Map of Thailand illustrating sampling collection sites for abalone (H. asinina, $H$. ovina and $H$. varia) used in this study. Dots represent geographic locations (excluding $H$. asinina from Indonesia and Philippines) for which at least one abalone species was sampled. Note that Samet Island (SAM) is located in Rayong (RAY) province. electrotransformed to E. coli XL1-BLUE (Dower et al., 1988). Recombinant clones were selected by the lacZ system following standard protocols (Maniatis et al., 1982). DNA sequences of 2-6 clones of each insert were examined from both directions using an automated DNA sequencer (Li-Cor, Lincoln, USA).

Species-specific PCR, sensitivity, and stability tests Twenty pairs of primers were designed using OLIGO 4.0. They were preliminarily tested for specificity against genomic DNA of the target $(N=12)$ and non-target species $(N=4)$ using PCR conditions that are described by Klinbunga et al. (2000), except that the annealing temperature was increased to $65^{\circ} \mathrm{C}$. Four pairs of primers (CUHA2, CUHA12, CUHO3, and CUHV1) were further examined against larger specimens $(N=216$, Table 1). The amplification product was electrophoretically analyzed through $1.6 \%$ agarose gels.

Sensitivity of the detection was examined against varying concentrations of the target DNA template (10 pg to $25 \mathrm{ng}$ ), using the same conditions as were used for the specificity test. The stability of CUHA2 and CUHA12 was tested against the poor genomic DNA template that was extracted from 10 individuals of the ethanol-preserved larvae (approximately $1 \mathrm{~mm}$ in size stored at $4^{\circ} \mathrm{C}$ for $\left.6 \mathrm{mo}\right)$ and frozen $\left(3 \mathrm{yr}\right.$ at $\left.-30^{\circ} \mathrm{C}\right)$, dried $\left(80^{\circ} \mathrm{C}\right.$ for $72 \mathrm{~h}$ and kept at room temperature for $2 \mathrm{wk}$ ), and boiled (10 min) $H$. asinina broodstock using the Chelex-based method (Walsh et al., 1994).

\section{Results and Discussion}

RAPD-PCR has widely been used for population genetic studies as well as the identification of molecular markers for various applications in several organisms (Heipel et al., 1998; Tassanakajon et al., 1998; Klinbunga et al., 2001). An 
Table 2. Population-, region-, and species-specific markers of tropical abalone in Thailand (H. asinina, H. ovina, and H. varia) revealed by RAPD analysis using primers OPB11, UBC101, UBC195, UBC197, and UBC271

\begin{tabular}{|c|c|c|c|}
\hline Species/population & Primer & $\begin{array}{l}\text { RAPD marker } \\
\text { (bp) }\end{array}$ & Name of Clones \\
\hline \multirow[t]{10}{*}{ H. asinina } & OPB11 & 1400 & pCUHA14 and pCUHA16 \\
\hline & & 800 & pCUHA17 and pCUHA18 \\
\hline & UBC101 & 1700 & pCUHA19 \\
\hline & & 1325 & pCUHA20 \\
\hline & & 590 & pCUHA13 \\
\hline & UBC195 & 760 & pCUHA4, pCUHA21 and pCUHA22 \\
\hline & UBC197 & 1400 & pCUHA23 \\
\hline & & 710 & pCUHA1 \\
\hline & UBC271 & 1000 & pCUHA24 \\
\hline & & 650 & pCUHA2, pCUHA3, pCUHA5, pCUHA6, pCUHA12 and pCUHA25 \\
\hline \multirow[t]{3}{*}{ /HATRAW } & UBC195 & 650 & pCUHA10 and pCUHA11 \\
\hline & UBC271 & 850 & pCUHA7 and pCUHA8 \\
\hline & & 450 & pCUHA9 \\
\hline \multirow[t]{3}{*}{ /HAPHIE } & UBC195 & 1000 & pCUHA26 \\
\hline & & 680 & pCUHA27 \\
\hline & UBC197 & 415 & pCUHA15 \\
\hline \multirow[t]{2}{*}{ H. ovina } & OPB11 & 475 & pCUHO1, pCUHO2 and pCUHO3 \\
\hline & UBC195 & 950 & pCUHO4 \\
\hline /Gulf of Thailand & UBC195 & 1650 & pCUHO5 \\
\hline \multirow[t]{3}{*}{ H. varia } & OPB11 & 690 & pCUHV4, pCUHV5, pCUHV6 and pCUHV7 \\
\hline & UBC195 & 700 & pCUHV1 and pCUHV2 \\
\hline & & 550 & pCUHV3 \\
\hline
\end{tabular}

analysis of the genetic diversity and population differentiation of $H$. asinina, $H$. ovina, and $H$. varia using PCR-RFLP of $16 \mathrm{~S}$ and 18S rDNA (Jarayabhand et al., 2002; Klinbunga et al., 2003) revealed a lack of genetic heterogeneity of $H$. asinina in Thailand $(\mathrm{P}>0.05)$. The analysis, however, did indicate significant genetic differences between $H$. asinina, $H$. ovina, and $H$. varia $(\mathrm{P}<0.0001)$. This suggested the possibility of identifying a large number of species-specific markers in $H$. asinina.

Among the 113 RAPD primers that were screened, 27 primers (OPA1, 2, 10, 15, 10, and 20, OPB11, 16, and 17, UBC101, 119, 160, 168, 174, 193, 195, 197, 200, 210, 220, 264, 267, 271, 272, 456, 457, and 459) yielded successful amplification results in three abalone. Five primers (OPB11, GTAGACCCGT; UBC101, GCGCCTGGAG; UBC195, GATCTCAGCG; UBC197, TCCCCGTTCC and UBC271, GCCATCAAGA) were selected for the identification of candidate species-specific (or population-specific) markers of each abalone.

In total, the 10, 2, and 3 fixed RAPD markers were found in $H$. asinina, $H$. ovina, and $H$. varia (Table 2 and Fig. 2 ). Three candidate population-specific RAPD markers were observed in $H$. asinina that originated from Talibong Island (HATRAW) and the Philippines (HAPHIE), respectively. In addition, an 1,650 bp RAPD marker that was generated from UBC195 was specifically found in $H$. ovina, originating from the Gulf

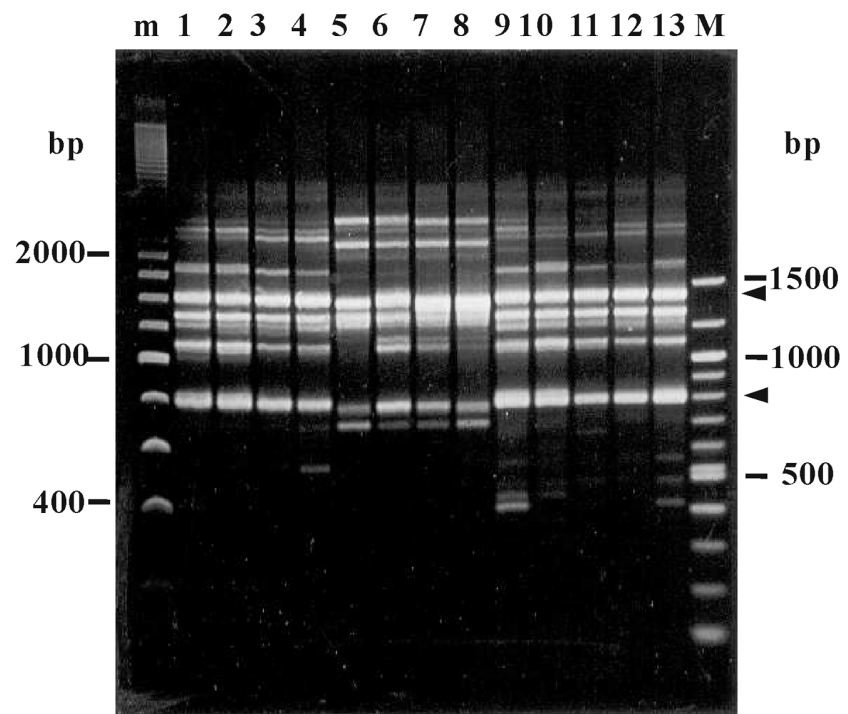

Fig. 2. An example of species-specific RAPD markers resulted from amplification of total DNA of $H$. asinina from HARAYE (lanes 1-4), HATRAW (lanes 5-8) and HACAMHE (lanes 9-13) with OPB11. Arrowheads indicate species-specific RAPD bands found in $H$. asinina. Lanes $\mathrm{M}$ and $\mathrm{m}$ are $100 \mathrm{bp}$ and $200 \mathrm{bp}$ DNA markers, respectively.

of Thailand (east), but not in the Andaman Sea sample (west). RAPD-PCR is sensitive to reaction conditions, including 
Table 3. Sequences of primers designed from population-specific (CUHA11) and species-specific RAPD markers of $H$. asinina, $H$. ovina, and $H$. varia

\begin{tabular}{ll}
\hline \multicolumn{1}{c}{ Primer } & \multicolumn{1}{c}{ Sequence } \\
\hline CUHA1 & F: 5'-GAATCCAACATGCGTCAAAG-3' \\
& R: 5'-CTGGAAACAATCGCAGGTCA-3' \\
& F: 5'-TTGTTCAGCATTCTGTGGCAGTTCT-3' \\
& R: 5'-CTTCTTTTTTGCTGACCCTTTGGAG-3' \\
CUHA4 & F: 5'-TCAGCGAAACCAACCAACAC-3' \\
& R: 5'-TTGGACGCAGCTATTCACAT-3' \\
CUHA11 & F: 5'-CCCCGAGGAGTATACAACTCTTCC-3' \\
& R: 5'-TCGAGTTCTTTCCACAATGCACC-3' \\
& FU: 5'-CTAATCCCACACAGCCATCACCAG-3' \\
& R: 5'- AAGAAGTGACGAAGAGGTAGGCAG-3' \\
CUHA13 & F: 5'-TGACCTGTGTTGAGACTCTACGGA-3' \\
& R: 5'-TGAGGGGAGATGGAGTAGCCGC-3' \\
& F: 5'-CGTGAAGACAGTTACTGAAAGTGG-3' \\
CUHA14 & R: 5'-ATCGTTTGTGTTATGTCTCCTCTG-3' \\
& F: 5'-GGGTATCTTCCCACAACAGC-3' \\
CUHO3 & R: 5'-GCACTTGCCTACATCCTTTCAC-3' \\
& F: 5'-CCCCTTGTTTCTCCTTCTTG-3' \\
CUHV1 & R: 5'-CGATGACGCAGGCGGTTTGA-3' \\
\end{tabular}

Table 4. Specificity of SCAR markers developed from population-specific or species-specific RAPD markers of $H$. asinina, $H$. ovina, and $H$. varia

\begin{tabular}{|c|c|c|c|c|c|c|c|}
\hline \multirow{2}{*}{ Primer } & \multirow{2}{*}{$\begin{array}{c}\text { Expected } \\
\text { product (bp) }\end{array}$} & \multicolumn{4}{|c|}{ H. asinina } & \multirow{2}{*}{ H. ovina } & \multirow{2}{*}{ H. varia } \\
\hline & & HACAMHE & HACAME & HATRAW & HAPHIE & & \\
\hline CUHA1-F/R & 292 & + & + & + & + & - & - \\
\hline CUHA2-F/R* & 168 & + & + & + & + & - & - \\
\hline CUHA3-F/R & 368 & NS & NS & NS & NS & NS & NS \\
\hline CUHA4-F/R & 290 & + & + & + & + & - & - \\
\hline CUHA5-F/R & 264 & + & + & + & + & + & + \\
\hline CUHA6-F/R & 103 & + & + & + & + & + & + \\
\hline CUHA7-F/R ${ }^{\mathrm{a}}$ & 554 & NS & NS & NS & NS & NS & NS \\
\hline CUHA8-F/R & 114 & NS & NS & NS & NS & NS & NS \\
\hline CUHA9-F/R & 142 & + & + & + & + & + & + \\
\hline CUHA10-F/R & 472 & NS & NS & NS & NS & NS & NS \\
\hline CUHA11-F/R & 417 & + & + & + & + & - & - \\
\hline CUHA12-F/R* & 312 & + & + & + & + & - & - \\
\hline CUHA13-F/R & 296 & + & + & + & + & - & - \\
\hline CUHA14-F/R & 473 & + & + & $\begin{array}{l}\text { a } 515 \text { bp } \\
\text { band }\end{array}$ & $\begin{array}{l}\text { a } 515 \text { bp } \\
\text { band }\end{array}$ & - & - \\
\hline CUHA15-F/R & 171 & + & + & + & + & + & + \\
\hline CUHO1-F/R & 414 & + & + & + & + & + & + \\
\hline CUHO2-F/R & 146 & + & + & - & - & $\begin{array}{c}+ \text { and a } 215 \text { bp } \\
\text { band }\end{array}$ & - \\
\hline CUHO3-F/R* & 328 & - & - & - & - & + & - \\
\hline CUHO4-F/R & 619 & NS & NS & NS & NS & NS & NS \\
\hline CUHV1-F/R* & 229 & - & - & - & - & - & + \\
\hline
\end{tabular}

Abbreviations: +, successful amplification with the expected product; -, no amplification product; NS, non-specific fragments obtained; *, primers further tested against a large sample size $(N=216)$; ${ }^{\text {, }}$, primers derived from HATRAW-specific RAPD markers; ${ }^{\mathrm{b}}$, primers derived from a HAPHIE-specific RAPD marker. 
(A)

GCCATCAAGA TGTGACATGT TCATGGGCAA CTAAAATGGA TTGAAAAAAC GTTACAAGTA TTTCACAAAC CTTTCCCTTT ATATATTTAT CCTGAATTAA ATTGAACTCT GCTGAAAAGT GGCATATCAT TAATCAAAAT ATAGACTGGT CAAACGTAAT GACTTCTTCA TGACTAAATA TAACTGAAGA TCAATGAACA AGGTAAATAT TGCTGTAAAC AATAAACAGG CACATGTTTC AAAACAATTC CACACAAAGG TAACTTTTGC TATTTGGTTA TGTAAACAAG CTGCAGAGCC CTCTTATGAA AGTTCACCTC CCAATGACTT CATTTATCAG TAATGTGTGA PCUHA2 - F

TCATATCCAG GATGCTTGTT CAGCATTCTG TGGCAGTTCT TGACAACTTG ATTTCAGTAC TGAATGAACA AGAAAGACTG ACAATTTTGT TCCATGTTGC CCCCAAGTTA TTCTTGACAG ATGTAGATTC TGCATTAGTG ACAAAGATTT PCUHA2 - $R$

TTATATATCT CCAAAGGGTC AGCAAAAAAG AAGTACAGTA TACCCTGGTG ATATCTTCCA CTGTTTGTCA GAGGGAACAT ATGGTACTGT ATCCAGACAC ACGTTATAAC CAGGGTGCAC TGTACATGCC CACATGGAAG CATGAATCAT GACAGTCAAT ACCATAACTG TATTTAATTT ACAAAAAAAA ATATATATGA TCTTGATGGC

(B)

GCCATCAAGA ATAAGCGTTA TGAAAACACT AACTCAAATC AGCCAAATAA TCCCACTAGT GATATCCTCT TTGGACTGAA GTTGTTTGTT TTAAGAGTAA PCUHA12 - F

GAGGATTATA AGGCATGCTC AACTGGGTTT GGTCGTTTAA CTAATCCCAC ACAGCCATCA CCAGCAGAGG GAATGATTTA CACATCTCAT TTTTTGTGCT TAATTATTGT GTTTGTTTTT TATTGAAAAA AAAATGAAAT GGGAGTGTGA ACAGCTTTAC AGTTTATATC GACACTGGTA CCATACAACA TACAATACAA AATTTAAATG CAGCATGTGT TGTCACATAT GTATATAAAT TTGAATTACA AACATGGGTA AAGCTTGAAG ACAATCTTAT ATGGAAATAG TGAATTATCT PCUHA12 - $R$ GATCTTGATA ATCTGTCCAT CTTAAGATCT GCCTACCTCT TCGTCACTTC TTCTTTTAGA TTTGATACTA AACATGATTG AAGAATTAAC CTCGTGGTTT GTCATATGAC AATCTTGTAT TAGAACTAGG TTAGGTTTAT TTACCTTTGT AAAGTGCATT ACACACATAC AAACTAACTG TATTAATATA GAGCATTTAT GAGAATGGTA TTGGTAAGAN AATTATTTTT GGTGTCTTGA TGGC

(C)

GATCTCAGCG CATACATAÀT CATATTCATT ACAAAGGATA TAACACCAGA AATGTCGCGC CATAATTCTT ATGACATACA GAAACCGGGG TAATTTTATC GGCATCGCTG CTATATAATT TCCCCTCCTG AAACTAATAG AATTTCCTAA PCUHV1 - $F$

TTGGGGETGT CATGGTAGGT TCTTTGCCTA ACCCCTTGTT TCTCCTTCTT GTCGACATGA AAAGACCACT TTATCAAATA TTTCATCCTC ACTTCATTCA CAATAATCTC TTGATGAGCC CAACATTCGT CTGAAACATC CGCACGAGGC AGCCGGAACC ACCAGGCTGT TGGCAACTTA TTCAAAGTCT CTTCTCTTCA CCCACAGAGA TTTTTTCACA ATACCAACAA ACAATATCCA TCAAACCGCC PCUHA1-R

TGCGTCATCG TTTGTACTCA AGCCTCAATC GAACTTCGCC AGTGCACTAT GGACGTGCTT CGACGGECGG CTGCAAACCT TTGCAACAAA ACCACCGATT CTAGAAGTTT CGTTCATTGC ATTCCCCCGA CAACTGCTAT TATAAAACAT TTAGTTAGTG TAATTGTAAC TGGTCTATCT CGAGTCTTCG TCTTCATTAC GGATAATTAG CCCGTCGCTG AGGGGGGCAC TTTCTACGCT CATCCATCCC GCTGAGATC

Fig. 3. Sequences of CUHA2 (A), CUHA12 (B) and CUHV1 (C). The locations and sequences of $H$. asinina- and $H$. varia-specific forward primers and those complementary to reverse primers are labeled in boldface and underlined.

the requirement of a good quality DNA template for consistent results. False negatives may possibly occur. We then converted the candidate species-specific (and populationspecific) RAPD fragments to sequence-characterized amplified region (SCAR) markers (Weising et al., 1995).

Twenty-two RAPD fragments were cloned (Table 2). Seventy-two clones were sequenced. Thirty-nine different sequences were found. These indicated that the RAPD 

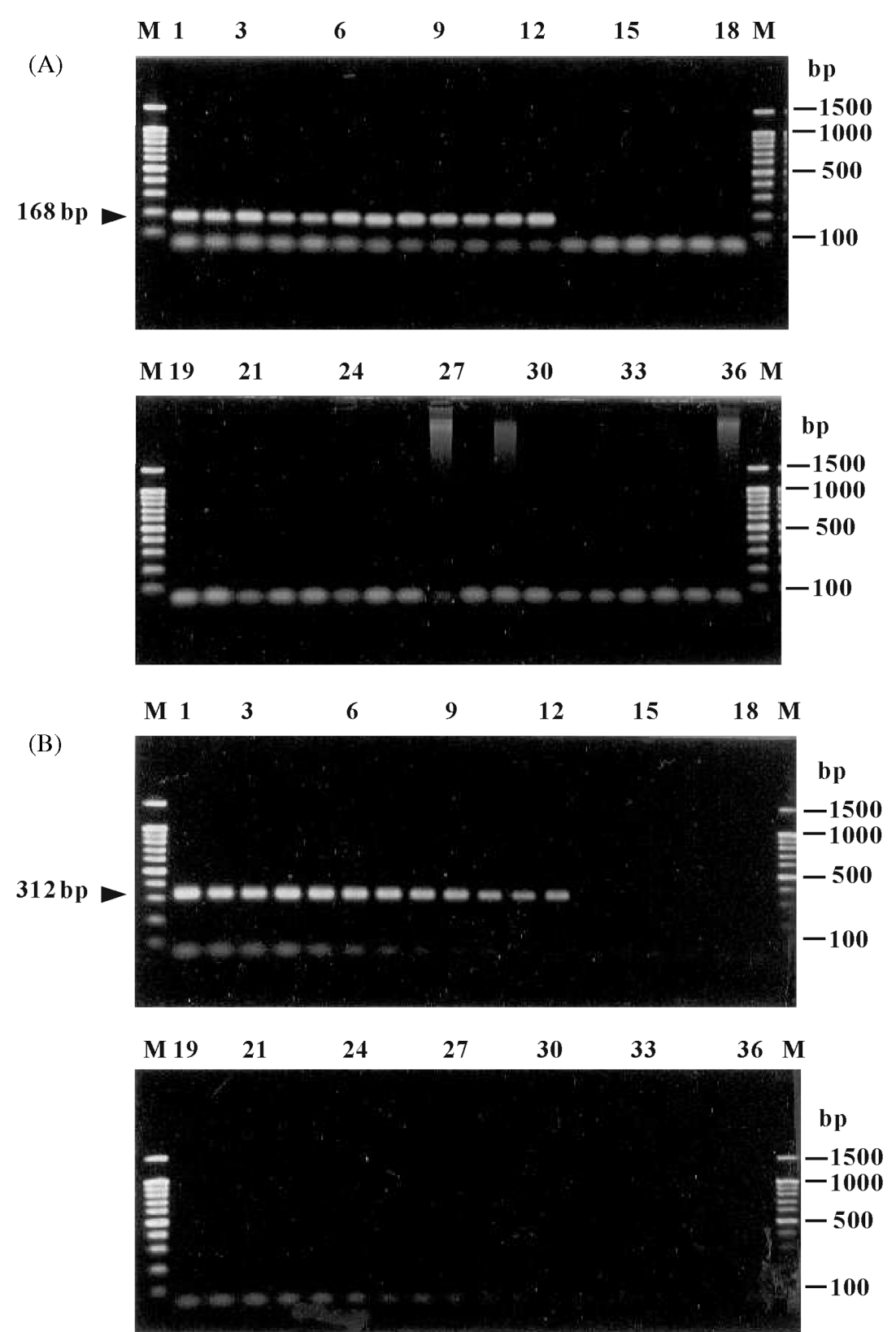

Fig. 4. Agarose gel elctrophoresis illustrating species-specificity of CUHA2 (A), CUHA12 (B) and CUHV1 (C) against genomic DNA of $H$. asinina (panels A, B and C, lanes 1-12), H. ovina (panels A, B and C, lane 13-24), and $H$. varia (panels A, B and C, lanes 2536). An 100 bp ladder (lanes M) was used as a DNA marker.

fragments represented co-migrating fragments that had different nucleotide sequences but similar sizes. Almost all of the RAPD markers (33/39 accounting for 85\%) were unknown sequences when compared with the data in the GenBank using Blast $N$ and Blast $X$ (E values $\left.>10^{-4}\right)$.

Twenty pairs of primers were designed from those unknown sequences (pCUHA1-pCUHA15, pCUHO1pCUHO4 and pCUHV1; Table 2). Originally, seven primer sets were designed for the development of population-specific markers in $H$. asinina. Four of these (CUHA3, CUHA7, CUHA8, and CUHA10) provided non-specific amplification results; CUHA9 and CUHA15 provided positive amplification in all of the abalone species and CUHA11 yielded a $H$. asinina-specific rather than a HATRAW-specific nature (Table 3). Therefore, population-specific SCAR markers were not found in $H$. asinina (Table 4).

In addition, the CUHA5, CUHA6 and CUHO1 primers also showed positive amplification bands in all three abalone species. We further tested the specificity of CUHA5 (264 bp) and CUHA6 (103 bp) against gastopods (the giant African snail, Achatina fulica, and the apple snails, Pomacea canaliculata, Pila ampullacea, P. angelica, P. pesmei, and P. 


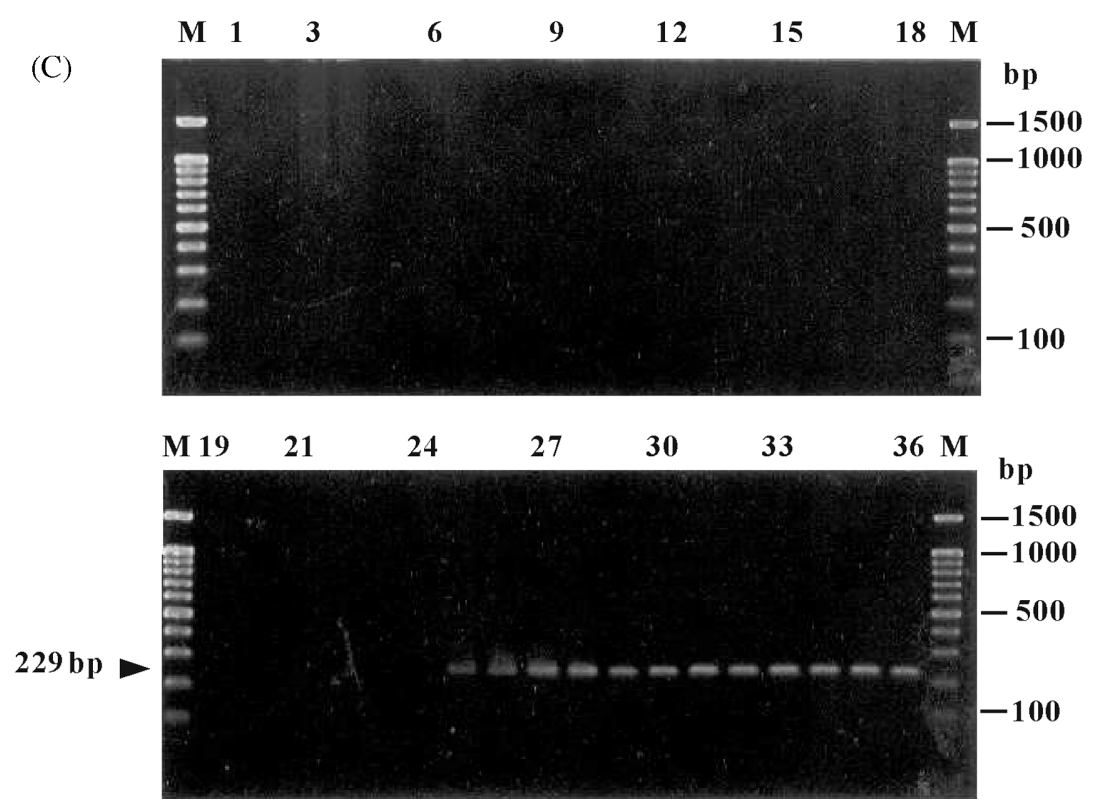

Fig. 4. Continued.

polita), oysters (Crassostrea belcheri, C. iredalei, Saccostrea cucullata, S. forskali, and Striostrea mytiloides), mussel (Perna viridis), and crustaceans (the black tiger prawn, Penaeus monodon and the giant freshwater prawn, Macrobrachium rosenbergii) (data not shown). These primers provided no positive amplification fragments in the nonabalone species and may be used as genus-diagnostic markers of abalone in this study.

Based on the preliminary screening, seven pairs of primers (CUHA1, CUHA2, CUHA4, CUHA11, CUHA12, CUHA13, and CUHA14) revealed species-specificity in $H$. asinine, while the CUHO3 and CUHV1 primers exhibited the expected product in $H$. ovina and $H$. varia, respectively (Tables 3 and 4). We did not select CUHA14 for the analysis against larger specimens of $H$. asinina because different sizes of the amplification products were observed (expected $473 \mathrm{bp}$ in HACAMHE and HACAME and a larger $515 \mathrm{bp}$ in HATRAW and HAPHIE, respectively).

Four sets of primers (CUHA2, CUHA12, CUHO3, and CUHV1, Fig. 3) were further examined against a large sample size of abalone $(N=216$, Table 1$)$. Species-specificity was observed from CUHA2, CUHA12, and CUHV1 (100\% without false positive/negative results, Fig. 4). Although CUHO3 yielded a strong amplification product in the target species (100\%), a very faint product was also observed in some individuals of $H$. asinina and $H$. varia, which suggests that problems may arise from the non-specific amplification of this primer pair (data not shown). No heterozygotes that exhibited two different sizes (alleles) of the amplification products were observed across the overall specimens that were analyzed by these primers. This implies the retention of a dominant segregated fashion of the original RAPD markers.

A limited sample size of $H$. varia was included in this study
( $N=29$ and 3 for HVPHAW and HVPHUW, respectively). This was due to a lack of $H$. varia that was found in our sampling sites. Accordingly, a $H$. varia-specific SCAR marker (229 bp from CUHV1) should be further tested against specimens that cover a larger geographic distribution of $H$. varia before practical implementation of this marker for the species-identification purpose.

The sensitivity of CUHA2, CUHA12, and CUHV1 was tested using different concentrations of the target DNA template $(10 \mathrm{pg}-25 \mathrm{ng})$. These primer pairs revealed a good correlation between the amount of DNA template and intensity of the PCR product. The detection sensitivity was approximately $100 \mathrm{pg}$ of the target DNA template for CUHA2 and CUHA12, but there was a greater sensitivity with CUHV1 (20 pg). The sensitivity levels of the species-specific PCR that was developed in this study were sufficient for the identification of the species-origins of abalone, beginning with the early development stages.

For rapid species-identification of $H$. asinina, the tedious and time-consuming phenol/chloroform extraction method was simplified to a rapid 5\% Chelex-based method. The positive fragment (168 bp) of CUHA2 was still consistently obtained from frozen, ethanol-preserved, dried and boiled specimens of $H$. asinina. All but a single dried specimen was successfully amplified by CUHA12 (Fig. 5).

In the present study, we demonstrate the successful development of species-specific SCAR markers in $H$. asinina and $H$. varia. These markers can be used to verify speciesorigins of various forms of abalone products from Thailand and prevent supplying incorrect abalone larvae for the culture industry. In the future, a large number of the remaining clones, possessing $H$. asinina-specific RAPD inserts, guarantee that additional SCAR markers could be developed if diagnostic 

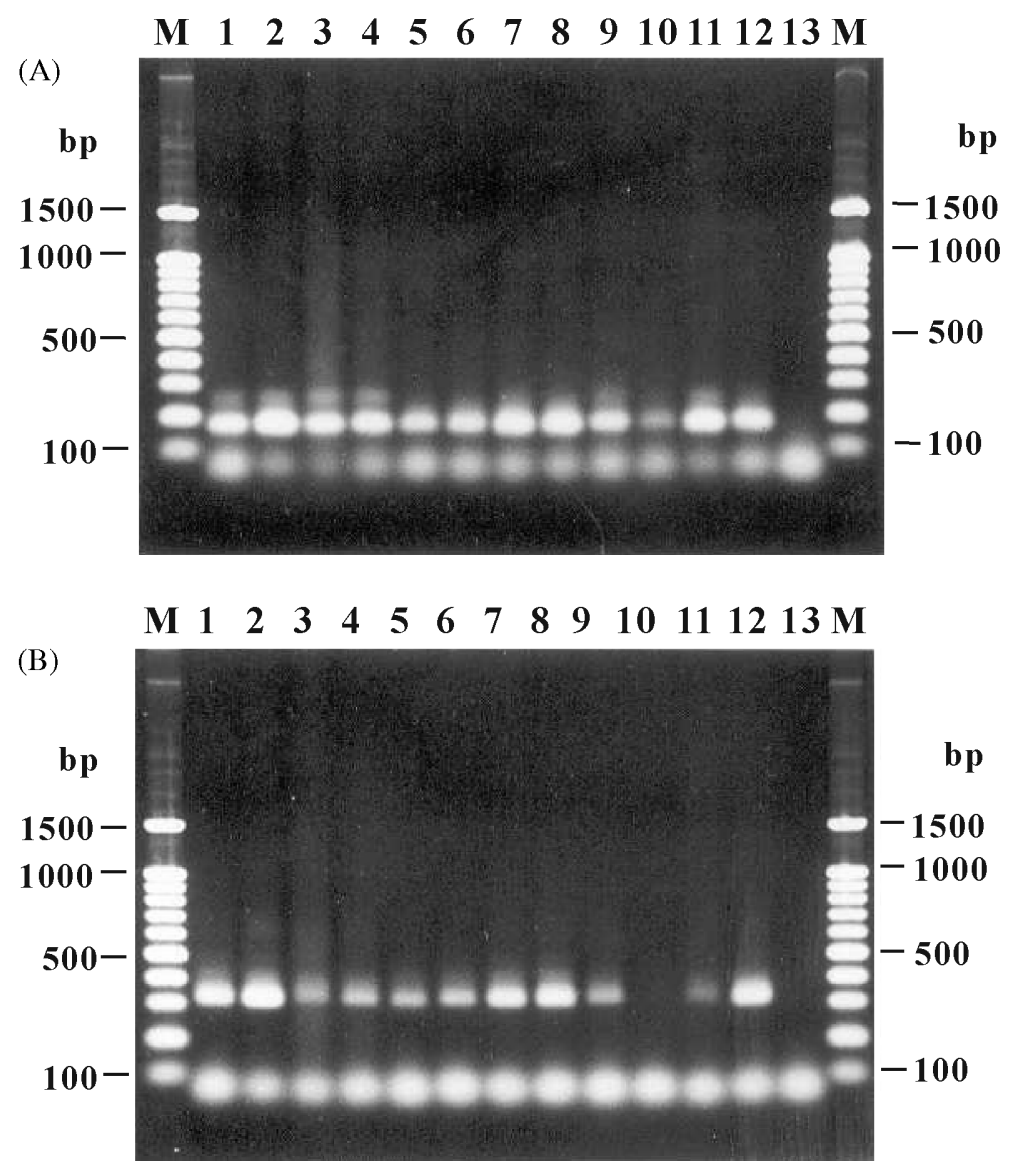

Fig. 5. Agarose gel elctrophoresis showing results from amplification of total DNA extracted with a phenol/chloroform (lanes 1-2) and a 5\% Chelex extraction methods (lanes 3-12) of frozen (lanes 1-4), ethanol-preserved larvae (lanes 5-8), dried (lanes 9-10) and boiled (11-12) broodstock of $H$. asinina with CUHA2 (panel A) and CUHA12 (panel B). Lanes 13 are negative controls (without DNA template). A $100 \mathrm{bp}$ ladder (lanes M) was used as a DNA marker.

markers that are described here fail to provide species-specific results when used to examine the species-origins of new populations of $H$. asinina.

Acknowledgments We thank the two anonymous referees for their useful comments. This work was supported by the Thailand Research Funds (TRF) Project, 4320015, that was awarded to SK and AT and was partially supported by JSPS (DNA sequencing). The Royal Golden Jubilee Ph.D. Program, TRF, granted a student fellowship to PA.

\section{References}

Avise, J. C. (1994) Molecular Markers, Natural History and Evolution, Chapman and Hall, London, UK.

Brown, L. D. (1995) Genetic evidence for hybridization between Haliotis rubra and H. laevigata. Mar. Biol. 123, 89-93.

Calvalho, G. R. and Hauser, L. (1994) Molecular genetics and the stock concept in fisheries. Rev. Fish Biol. Fisheries 4, 326-350.

Dower, W. J., Miller, J. F. and Ragsdale, C. W. (1988) High efficiency transformation of $E$. coli by high voltage electroporation. Nucleic Acids Res. 16, 612-617.

Fritsch, P., Hanson, M. A., Spore, C. D., Pack, P. E. and Rieseberg, L. H. (1993) Constancy of RAPD primer amplification strength among distantly related taxa of flowering plants. Plant Mol. Biol. Rep. 11, 10-20.

Gordon, H. R. (2000) World abalone supply, markets and pricing: historical, current and future prospectives. $4^{\text {th }}$ International Abalone Symposium, 6-11 February, 2000, University of Cape Town, Cape Town, South Africa.

Hadrys, H., Balick, M. and Schierwater, B. (1992) Applications of random amplified polymorphic DNA (RAPD) in molecular ecology. Mol. Ecol. 1, 55-63.

Heipel, D. A., Bishop, J. D. D., Brand, A. R. and Thorpe, J. P. (1998) Population genetic differentiation of the great scallop Pecten maximus in western Britain investigated by randomly amplified polymorphic DNA. Mar. Ecol. Prog. Ser. 162, 163171.

Jarayabhand, P. and Paphavasit, N. (1996) A review of the culture of tropical abalone with special reference to Thailand. Aquaculture 140, 159-168.

Jarayabhand, P., Pripue, P., Klinbunga, S. and Tassanakajon, A. (2002) Identification of species-diagnostic markers of abalone in Thailand using PCR-RFLP of 16S rDNA. Fisheries Sci. 68 
(suppl II), 1091-1094.

Klinbunga, S., Sodsuk, S., Penman, D. J. and McAndrew, B. J. (1996) An improved protocol for total DNA isolation and visualization of mtDNA RFLP(s) in the giant tiger prawn, Penaeus monodon. Thai J. Aquat. Sci. 3, 36-41.

Klinbunga, S., Ampayup, P., Tassanakajon, A., Jarayabhand, P. and Yoosukh, W. (2000) Development of species-specific markers of the tropical oyster (Crassostrea belcheri) in Thailand. Mar. Biotechnol. 2, 476-484.

Klinbunga, S., Ampayup, P., Tassanakajon, A., Jarayabhand, P. and Yoosukh, W. (2001) Genetic diversity and molecular markers of cupped oysters (Genera Crassostrea, Saccostrea and Striostrea) in Thailand revealed by RAPD analysis. Mar. Biotechnol. 3, 133-144.

Klinbunga, S., Pripue, P., Khamnamtong, N., Tassanakajon, A., Jarayabhand, P. and Menasveta, P. (2003) Genetic diversity and molecular markers of the tropical abalone (Haliotis asinina) in Thailand. Mar. Biotechnol. (in press).

Lee, Y. H. and Vacquier, V. D. (1995) Evolution and systematics in Haliotidae (Mollusca: Gastropoda): inferences from DNA sequences of sperm lysin. Mar. Biol. 124, 267-278.

Maniatis, T., Frisch, E. F., and Sambrook, J. (1982) Molecular Cloning: A Laboratory Manual, Cold Spring Harbor Laboratory Press, New York, USA.

Muchmore, M. E., Moy, G. W., Swanson, W. J. and Vacquier, V. D. (1998) Direct sequencing of genomic DNA for characterization of a satellite DNA in five species of Eastern Pacific abalone. Mol. Mar. Biol. Biotechnol. 7, 1-6.

Naganuma, T., Hisadome, K., Shiraishi, K. and Kojima, H. (1998) Molecular distinction of two resemblant abalones, Haliotis discus discus and Haliotis discus hannai by $18 \mathrm{~S}$ rDNA sequences. J. Mar. Biotechnol. 6, 59-61.

Nateewathana, A. and Bussarawit, S. (1988) Abundance and distribution of abalone along the Andaman Sea coast of Thailand. Kasetsart J. (Natural Science) 22, 8-15.
Singhagraiwan, T. and Doi, M. (1993) Seed production and culture of a tropical abalone, Haliotis asinina Linne. Department of Fisheries, Ministry of Agriculture and Cooperatives, Thailand.

Sweijd, N. A., Bowie, R. C. K., Lopata, A. L., Marinaki, A. M., Harley, E. H. and Cook, P. A. (1998) A PCR technique for forensic, species-level identification of abalone tissue. $J$. Shellfish Res. 17, 889-895.

Tassanakajon, A., Pongsomboon, S., Jarayabhand, P., Klinbunga, S. and Boonsaeng, V. (1998) Genetic structure in wild populations of the black tiger shrimp (Penaeus monodon) using randomly amplified polymorphic DNA analysis. J. Mar. Biotechnol. 6, 249-254.

Tookvinas, S., Leknim, V., Donyadol, Y., Predalampabut, Y. and Paengmark, P. (1986) A survey of species and distribution of abalone (Haliotis spp.) in Surat Thani, Nakhon Si Thammarat and Songkla. Tech. Rep. No. 1/1986 NICA, 16 pp.

Vacquier, V. D., Carner, K. R. and Stout, C. D. (1990) Speciesspecific sequences of abalone lysin, the sperm protein that creates a hole in the egg envelope. Proc. Natl. Acad. Sci. USA 87, 5792-5796.

Walsh, P. S., Metzger, D. A. and Higuche, R. (1994) Chelex ${ }^{\circledR} 100$ as a medium for simple extraction of DNA for PCR-based typing from forensic materials. Biotechniques 10, 506-513.

Weising, K., Nybom, H., Wolf, K. and Meyer, W. (1995). DNA Fingerprinting in Plant and Fungi. CRC Press, Boca Raton, USA.

Welsh, J. and McClelland, M. (1990) Fingerprinting genomes using PCR with arbitrary primers. Nucleic Acids Res. 18, 72137218.

Williams, J. G. K., Kubelik, A. R., Livak, K. J., Rafalski, J. A. and Tingey, S. V. (1990). DNA polymorphisms amplified by arbitrary primers are useful as genetic markers. Nucleic Acids Res. 18, 6531-6535. 